\title{
Computational analysis of the effect of superparamagnetic nanoparticle properties on bioheat transfer in magnetic nanoparticle hyperthermia
}

\author{
Frederik Soetaert, Luc Dupré, and Guillaume Crevecoeur
}

\begin{abstract}
Magnetic nanoparticle hyperthermia is an alternative cancer treatment where magnetic nanoparticles are delivered to the tumor. The magnetization response due to an alternating magnetic field causes the magnetic nanoparticles to act as a heating power source and thermally damage the tumor cells. The heating capability of magnetic nanoparticles depends on their relaxation losses and thus on their material properties. Heat transfer in biological tissues on the other hand depends on the material properties of the biological tissue, as well as the spatial distribution of the magnetic nanoparticles. This paper presents an efficient numerical calculation method for assessing the temperature distribution in biological tissue when considering magnetic nanoparticles and biological tissues with specified material properties. We firstly investigate the effect of polydisperse magnetite and maghemite magnetic nanoparticles distributions on the temperature. We furthermore study the influence of the spatial spread of the magnetic nanoparticles inside the tumor on the temperature distribution and the associated thermal damage. The proposed numerical methodology is able to predict temperature elevations in biological tissues due to magnetic nanoparticle hyperthermia and can constitute an important component for model-based optimization of magnetic nanoparticle hyperthermia.
\end{abstract}

Index Terms-magnetic nanoparticles, hyperthermia, magnetic properties, specific loss power

\section{INTRODUCTION}

$\mathbf{M}$ AGNETIC nanoparticle hyperthermia (MNH) is a promising cancer treatment. Before the treatment, magnetic nanoparticles (MNPs) are delivered either systemically or by direct injection to the tumoral tissue. When exposed to an alternating magnetic field (AMF), different processes of magnetization reversal arise and lead to magnetic losses that result in heat [1]. A cell-killing effect is observed when the local temperature in a biological tissue resides between 41 and $46^{\circ} \mathrm{C}$ for a period of time [2]. Because biological tissue is only weakly diamagnetic, MNH offers the potential for a precise and tissue-specific thermal dose delivery [3].

The heating efficiency of magnetic nanoparticles is usually expressed in terms of specific loss power (SLP) with unit watts per gram of iron. Theoretical studies have attempted to predict this heating efficiency as a function of the applied magnetic field amplitude and frequency. The linear response

F. Soetaert is a Ph.D. fellow of the Research Foundation - Flanders (FWO).

F. Soetaert, L. Dupré, and G. Crevecoeur are with the Department of Electrical Energy, Systems and Automation, Ghent University, Ghent, Belgium, e-mail: frederik.soetaert@ugent.be

978-1-4673-9575-5/16/\$31.00 @2016 IEEE theory (LRT) is a popular theoretical framework describing the hysteresis loop area of non-interacting single domain MNPs when the magnetization is linear with the applied external field [4], [5]. LRT is suitable to predict the heating efficiency of MNPs in the superparamagnetic regime [5]. In this computational study, we will use a modified version of the linear response theory in order to account for a non-negligible anisotropy [5].

The theoretically predicted heating source of the superparamagnetic nanoparticles acts as an input to the bioheat transfer model of Pennes [6]. The aim of this numerical study is to analyze the output of the bioheat transfer model (i.e. the temperature variations as well as the thermal damage in biological tissues) when applying an AMF to magnetite and maghemite magnetic nanoparticles. This paper proposes an efficient computational model to investigate the effect of superparamagnetic nanoparticles properties on the heat transfer in magnetic nanoparticle hyperthermia, offering the potential of model-based optimization.

\section{SPECIFIC LOSS POWER}

Linear response theory describes the dynamic response of an ensemble of non-interacting single domain MNPs to a timevarying external magnetic field having a certain amplitude $H_{a}$ and frequency $f$ (with associated angular frequency $\omega$ ). The magnetization response of the MNPs to the externally applied magnetic field $H(t)=H_{a} \cos (\omega t)$ follows

$$
M(t)=H_{a}\left[\chi^{\prime} \cos (\omega t)+\chi^{\prime \prime} \sin (\omega t)\right]
$$

with the complex susceptibility being

$$
\chi=\chi^{\prime}-j \chi^{\prime \prime}
$$

The susceptibility $\chi$ is assumed to be independent of the applied magnetic field. Consequently, this theory is commonly referred to as the linear response theory [5], [3]. The imaginary part of (2) is responsible for the phase lag between the externally applied magnetic field and the response magnetization of the MNP and hence creating relaxation losses. The imaginary susceptibility's frequency-dependence can further be detailed as [4], [5]

$$
\chi^{\prime \prime}=\frac{\omega \tau}{1+(\omega \tau)^{2}} \chi_{m, 0}
$$


with the static susceptibility $\chi_{m, 0}$ that can be regarded as the chord susceptibility corresponding to the Langevin equation:

$$
\chi_{m, 0}=\chi_{m, i} \frac{3}{\xi}\left[\operatorname{coth}(\xi)-\frac{1}{\xi}\right]
$$

with $\xi=\frac{\mu_{0} M_{s} H_{a} V_{m}}{k_{B} T} \cdot \chi_{m, i}$ is the initial susceptibility determined from differentiation of the Langevin equation:

$$
\chi_{m, i}=\frac{\mu_{0} \phi M_{s}^{2} V_{m}}{3 k_{B} T}
$$

with $\mu_{0}$ the vacuum permeability, $M_{s}$ the saturation magnetization and $V_{m}=\frac{4}{3} \pi r^{3}$ the magnetic volume of a spherical MNP with radius $r, k_{B}$ the Boltzmann constant and $T$ the temperature. $\phi$ is the ferrofluid volume fraction. Carrey et al. [5] propose a modification of the traditional linear response theory to account for the anisotropy of MNPs. A phenomenological fit is provided for the initial susceptibility (5), i.e.

$$
\chi_{m, i}=\frac{\mu_{0} \phi M_{s}^{2} V_{m}}{3 k_{B} T} \cdot\left[3-\frac{2}{1+\left(\frac{K V_{m}}{k_{B} T} / 3.4\right)^{1.47}}\right]
$$

with $K$ being the anisotropy constant of the MNP.

The relaxation time $\tau$ in (3) consists of both Néel and Brown relaxation phenomena with $\tau_{N}$ and $\tau_{B}$ as the respective relaxation constants. The global relaxation constant can be expressed as

$$
\tau=\left(\frac{1}{\tau_{B}}+\frac{1}{\tau_{N}}\right)^{-1}=\frac{\tau_{B} \tau_{N}}{\tau_{B}+\tau_{N}}
$$

and is an important factor for relaxation losses of superparamagnetic MNP with

$$
\tau_{N}=\tau_{0} \exp \left(\frac{K V_{m}}{k_{B} T}\right)
$$

and

$$
\tau_{B}=\frac{3 \eta V_{h}}{k_{B} T}
$$

$\tau_{0}$ in (8) is the inverse of the attempt frequency [7] and has typical values in the order of nanoseconds. $\eta$ and $V_{h}$ in (9) are the solvent viscosity and the hydrodynamic volume of the magnetic nanoparticles respectively. When assuming a shell thickness $\delta, V_{h}=4(r+\delta)^{3} / 3 \pi$.

The volumetric power loss $P\left(W / m^{3}\right)$ can be derived by multiplying the frequency with the hysteresis loop area:

$$
P=\pi \mu_{0} \chi_{m, 0} H_{a}^{2} f \frac{\omega \tau}{1+(\omega \tau)^{2}}
$$

For further details related to the derivation, we refer to [4], [5]. In magnetic nanoparticle hyperthermia literature, we usually refer to the specific loss power (SLP) instead of the volumetric power loss $P$, i.e.

$$
S L P_{\mathrm{LRT}}=\frac{P}{\rho \phi}
$$

with $\rho$ the mass density of the magnetic material in $g / \mathrm{m}^{3}$ such that the dimension of SLP is $W / g_{\mathrm{Fe}}$.

When considering a magnetic nanoparticle distribution, the individual sizes of each MNP will not be identical due to the production process [8]. In fact, the MNPs radii mostly follow a log-normal particle size distribution. We will therefore consider such polydisperse MNP populations that follow the log-normal probability density function

$$
p(r)=\frac{1}{\sigma_{r} r \sqrt{2 \pi}} \exp \left[\frac{-\ln ^{2}\left(r / \mu_{r}\right)}{2 \sigma_{r}^{2}}\right]
$$

with $\mu_{r}$ and $\sigma_{r}$ being the mean and standard deviation of the MNP radii. The associated specific loss power (11) has to be weighted according to (12) [8]:

$$
<S L P_{\mathrm{LRT}}>=\int_{0}^{\infty} S L P_{\mathrm{LRT}}(r) \cdot p(r) \mathrm{d} r,
$$

\section{BIOHEAT TRANSFER}

Heat transfer is the transfer of energy resulting from differences in temperature. The temperature variation in time and space can be described by the biological heat transfer model of Pennes [6]:

$$
\rho_{t} c_{p} \frac{\partial T}{\partial t}=\nabla \cdot(k \nabla T)-\omega_{b} \rho_{b} c_{b}\left(T-T_{b}\right)+P_{\mathrm{MNP}}
$$

consisting of the biological tissue's mass density $\rho_{t}$ and specific heat capacity $c_{p}$. The right hand side consists of the heat sources and sinks. The first term is related to the heat conduction with thermal conductivity $k$. The second term is the heat sink due to blood perfusion (with blood temperature $T_{b}=37^{\circ} \mathrm{C}$ where we assume that the heat transfer takes place in the capillary bed, i.e. we assume that no large blood veins are present in the considered volume). $\omega_{b}$ is the blood perfusion rate, $\rho_{b}$ is the blood mass density, and $c_{b}$ is the specific heat capacity of blood. The heat source originating from the magnetic nanoparticles $P_{\text {MNP }}$ can be related to the specific loss power (13) when considering magnetic nanoparticles with a log-normal radius distribution (with associated $\mu_{r}$ and $\sigma_{r}$ ).

We moreover consider that the magnetic nanoparticles are normally distributed in space (with coordinates $x, y, z$ ), following the probability density function $f_{\mathrm{MNP}}$, yielding the following heat power source:

$$
P_{\mathrm{MNP}}(x, y, z)=m_{\mathrm{Fe}} \cdot\left\langle S L P_{\mathrm{LRT}}>\cdot f_{\mathrm{MNP}}(x, y, z)\right.
$$

with

$$
f_{\mathrm{MNP}}(x, y, z)=\frac{1}{\sigma_{\text {spread }}^{3}(2 \pi)^{3 / 2}} \exp \left[-\frac{x^{2}+y^{2}+z^{2}}{2 \sigma_{\text {spread }}^{2}}\right]
$$

The spread of magnetic nanoparticles is described by $\sigma_{\text {spread }}$. The percentage of magnetic nanoparticles situated in the tumor is denoted by

$$
p_{t}=\int_{0}^{r_{\text {bound }}} p(r) \mathrm{d} r
$$

with $r_{\text {bound }}$ being the outer radius of the considered spherical tumor. By increasing the spread $\sigma_{\text {spread }}$, the percentage $p_{t}$ will decrease.

Equation (14) is solved by means of an accurate and efficient finite element implementation following [9]. The reduction from $3 \mathrm{D}$ to $1 \mathrm{D}$ is possible due to the assumed spherically symmetrical geometry and symmetrical spatial distribution of MNPs (16). The meshing is performed using Gmsh [10] and the numerical finite element calculations are performed using 
GetDP [11]. The time-stepping is carried out by a CrankNicholson scheme with fixed time step. In contrast to [9], we develop a computational model that does not have the SLP as a given heat source value but where the SLP is now numerically determined by means of (13).

Due to the elevated temperatures during the treatment, the biological tissue becomes thermally damaged. This thermal damage can be modeled by an Arrhenius model [12]

$$
\alpha=\exp \left[-\int_{0}^{t} A e^{\left(-\frac{\Delta E_{a}}{R \cdot T\left(\mathbf{r}, t^{\prime}\right)}\right)} \mathrm{d} t^{\prime}\right]
$$

with the gas constant $R$, frequency factor $A$ and activation energy barrier $\Delta E_{a}$. The temperature evolution at a certain point in space thus specifies the survival fraction $\alpha$, i.e. the concentration of living cells compared to its initial concentration.

\section{RESUlTS AND DISCUSSION}

The assumed alternating magnetic field has a fixed amplitude $H_{a}=10 \mathrm{kA} / \mathrm{m}$ and frequency $f=200 \mathrm{kHz}$. Two types of superparamagnetic nanoparticles are considered in our study, i.e. magnetite and maghemite nanoparticles with magnetic material properties listed in Table I. The Néelian (9) and Brownian (8) relaxation losses can be calculated using the additional data in that table for a given radius $r$ of magnetic nanoparticles. SLP calculations are performed in this study at $T=37^{\circ} \mathrm{C}$. We additionally assume that the macroscopic tissue temperature does not affect the relaxation losses. For a study on the temperature dependence of magnetic nanoparticles properties, we refer the reader to [13]. The bioheat transfer equation (14) and the thermal damage (18) are calculated with the constants provided in Table II. In this study, we assume a 1 $\mathrm{cm}^{3}$ spherical tumor (associated $r_{\text {bound }}=6.204 \mathrm{~mm}$ ) within a spherical liver of radius $2 \mathrm{~cm}$. The time stepping is performed using a fixed time step of $0.1 \mathrm{~s}$.

TABLE I

MAGNETIC NANOPARTICLE MATERIAL PROPERTIES

\begin{tabular}{|c|c|c|}
\hline & Symbol (units) & Value \\
\hline \hline Magnetite & & \\
\hline & $K\left(\mathrm{~kJ} / \mathrm{m}^{3}\right)$ & 23 \\
& $M_{s}(\mathrm{kA} / \mathrm{m})$ & 446 \\
\hline Maghemite & & \\
& $K\left(\mathrm{~kJ} / \mathrm{m}^{3}\right)$ & 4.7 \\
& $M_{s}(\mathrm{kA} / \mathrm{m})$ & 414 \\
\hline \hline & $\rho\left(\mathrm{kg} / \mathrm{m}^{3}\right)$ & 5260 \\
& $\eta\left(\mathrm{Ns} / \mathrm{m}^{2}\right)$ & 0.0009 \\
& $\delta(\mathrm{nm})$ & 2 \\
& $m_{\mathrm{Fe}}(\mathrm{mg})$ & 5 \\
\hline
\end{tabular}

Figure 1 shows the temperature variation both in the center of the tumor and averaged over the $1 \mathrm{~cm}^{3}$ tumor volume for three different spatial distributions (16), i.e. $p_{t}=95 \%, 80 \%$ and $50 \%$ of MNPs situated in the tumor. The corresponding $\sigma_{\text {spread }}$ values are given in Table III. We have a polydisperse collection of magnetite nanoparticles with $\mu_{r}=7 \mathrm{~nm}$ and $\sigma_{r}=0.01$. This $\mu_{r}$ corresponds with the maximal SLP as function of $\mu_{r}$, namely $S L P_{\mathrm{LRT}}=165.9 \mathrm{~W} / g_{\mathrm{Fe}}$. This figure illustrates that although the temperature rises sufficiently high
TABLE II

HEAT TRANSFER AND BIOLOGICAL TISSUE PROPERTIES

\begin{tabular}{|c|c|}
\hline Symbol (units) & Value \\
\hline \hline$\rho_{t}\left(\mathrm{~kg} / \mathrm{m}^{3}\right)$ & 1060 \\
$c_{p}\left(\mathrm{~J} /\left(\mathrm{kg} \mathrm{K}^{3}\right)\right.$ & 3500 \\
$k(\mathrm{~W} /(\mathrm{m} \mathrm{K}))$ & 0.53 \\
$\omega_{b}\left(\mathrm{~s}^{-1}\right)$ & 0.0064 \\
$\rho_{b}\left(\mathrm{~kg} / \mathrm{m}^{3}\right)$ & 1000 \\
$c_{b}(\mathrm{~J} /(\mathrm{kg} \mathrm{K}))$ & 4180 \\
\hline$A$ & $2.984 \cdot 10^{80}$ \\
$\Delta E_{a}$ & $5.064 \cdot 10^{5}$ \\
\hline$r_{\text {bound }}(\mathrm{mm})$ & 6.204 \\
\hline
\end{tabular}

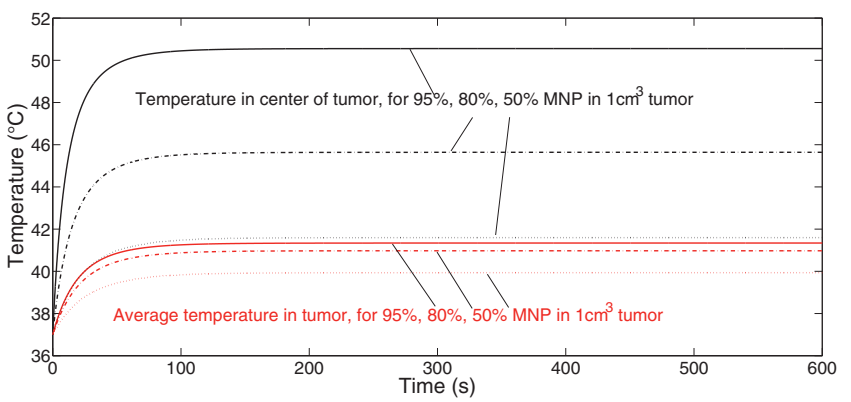

Fig. 1. Temperature variation for magnetite MNPs $\left(\mu_{r}=7 \mathrm{~nm}\right.$ and $\sigma_{r}=$ 0.01 ) subjected to $H_{a}=10 \mathrm{kA} / \mathrm{m}$ and $f=200 \mathrm{kHz}$ in the center of the tumor (black) and averaged over the tumor (red) for three different $\sigma_{\text {spread }}$.

in the center of the tumor, the average rise is limited in the tumor. Furthermore, the magnitude of the temperature rise significantly depends on the spatial spread of MNPs, highlighting the importance of obtaining a sufficient amount of MNPs in the tumor. We observe the onset of an equilibrium state around 200 s (i.e. $\frac{\partial T}{\partial t} \approx 0$ ). Figure 2 depicts the survival fraction in the center of the tumor (left) and averaged over the tumor volume (right), analogous to figure 1. This figure illustrates the effect of the spatial spread of magnetic nanoparticles on the survival fraction values. When considering the center of the tumor, virtually no cells survive if $p_{t}=95 \%$. If $p_{t}=80 \%$, only $20 \%$ of the cells survive after $600 \mathrm{~s}$. However, if one looks at the average survival fraction in the tumor, maximum $20 \%$ of the cells are thermally damaged $\left(p_{t}=95 \%\right)$.

TABLE III

CONSIDERED SPATIAL SPREAD OF MAGNETIC NANOPARTICLES AND CORRESPONDING PERCENTAGE OF MAGNETIC NANOPARTICLES IN THE TUMOR

\begin{tabular}{|c|c|}
\hline$\sigma_{\text {spread }}(\mathrm{m})$ & $p_{t}(\%)$ \\
\hline \hline 0.00222 & 95 \\
0.00290 & 80 \\
0.00405 & 50 \\
\hline
\end{tabular}

Further simulations were performed for magnetite and maghemite particles where the mean radius $\mu_{r}$ of the polydisperse MNP population (with fixed $\sigma_{r}=0.01$ ) is varied. We post-processed the results of the temperature and survival fraction at $200 \mathrm{~s}$, when equilibrium was achieved. The spatial spread of magnetic nanoparticles is again varied. Figures 3 and 4 show the results for the temperatures and survival fraction respectively. 

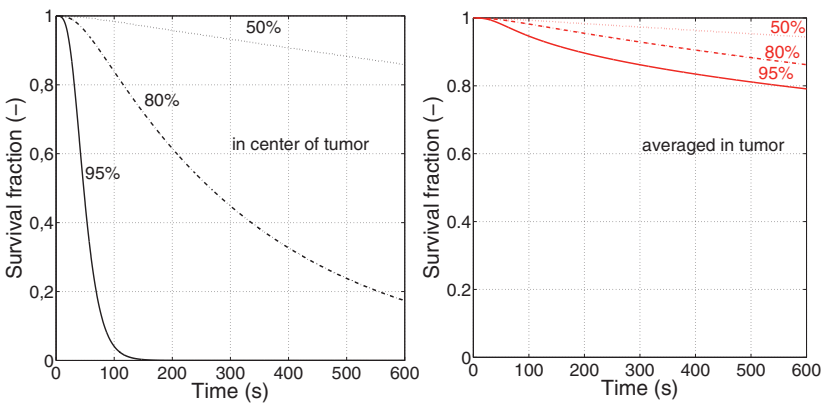

Fig. 2. Survival fraction variation for magnetite MNPs $\left(\mu_{r}=7 \mathrm{~nm}\right.$ and $\left.\sigma_{r}=0.01\right)$ subjected to $H_{a}=10 \mathrm{kA} / \mathrm{m}$ and $f=200 \mathrm{kHz}$ in the center of the tumor (left) and averaged over the tumor (right) for three different $\sigma_{\text {spread}}$.

Magnetite nanoparticles with a mean radius $\mu_{r}=7 \mathrm{~nm}$ achieve the highest temperature after $200 \mathrm{~s}$. When decreasing the amount of normally distributed MNPs in the tumor, the temperature in the center decreases (figure 1). It clearly indicates the importance of incorporating the spatial distribution and thus the spatial spread in the heat transfer calculations. One can also observe that the ratio of the temperature in the center and the temperature at the boundary of the tumor (i.e. at distance $r_{\text {bound }}$ ) decreases for decreasing $p_{t}$. The same trends hold for maghemite nanoparticles where maximum temperatures are reached around $\mu_{r}=11.5 \mathrm{~nm}$. The obtained temperatures are higher compared to magnetite MNPs because they generate higher $\left\langle S L P_{\mathrm{LRT}}>\right.$ values, confirming the results in [8]. Nonetheless, therapeutic effects $\left(T>41^{\circ} \mathrm{C}\right)$ can be observed in both types of MNPs. However, magnetite nanoparticles achieve these therapeutic temperatures in a larger radius range than maghemite nanoparticles. When $p_{t}=95 \%$ for instance, magnetite nanoparticles with a mean radius between $\mu_{r} \in[6.1-13.9] \mathrm{nm}$ have a maximum temperature above $41^{\circ} \mathrm{C}$. Maghemite nanoparticles only have a limited radius range to have a therapeutic effect: $\mu_{r} \in[9.9-13.6] \mathrm{nm}$. At the boundary of the tumor, the temperature is somewhat lower for $p_{t}=95 \%\left(39.6^{\circ} \mathrm{C}\right)$ than for $p_{t}=80 \%\left(40.5^{\circ} \mathrm{C}\right)$ and $p_{t}=50 \%\left(40.2^{\circ} \mathrm{C}\right)$. The average temperature in healthy tissue remains at the baseline temperature $\left(37^{\circ} \mathrm{C}\right)$, mainly due to the blood perfusion term in the heat transfer equation (14), indicating that no healthy cells will be thermally damaged.

The survival fraction of the cells in the center of the tumor is zero after $200 \mathrm{~s}$ for magnetite particles with $\mu_{r}=7 \mathrm{~nm}$ or maghemite particles with $\mu_{r}=11.5 \mathrm{~nm}$, spatially distributed with $p_{t}=95 \%$ of particles located in the tumor. However, due to the limited temperatures at the boundary of the tumor, the survival fraction remains close to 1 there. The heat transfer simulations clearly confirm that the challenge is to achieve a decrease in survival fraction in the center as well as at the tumor boundary. When increasing $H_{a}$ or the dose $m_{\mathrm{Fe}}$, the $P_{\text {MNP }}$ increases, yielding higher temperatures and decreasing survival fraction. Temperatures in the center of the tumor may reach unwanted high temperatures and almost no temperature rise at the boundary of the tumor. The results presented here suggest the importance of incorporating the spatial spread of the particles in the analysis as well as the mean radius of the
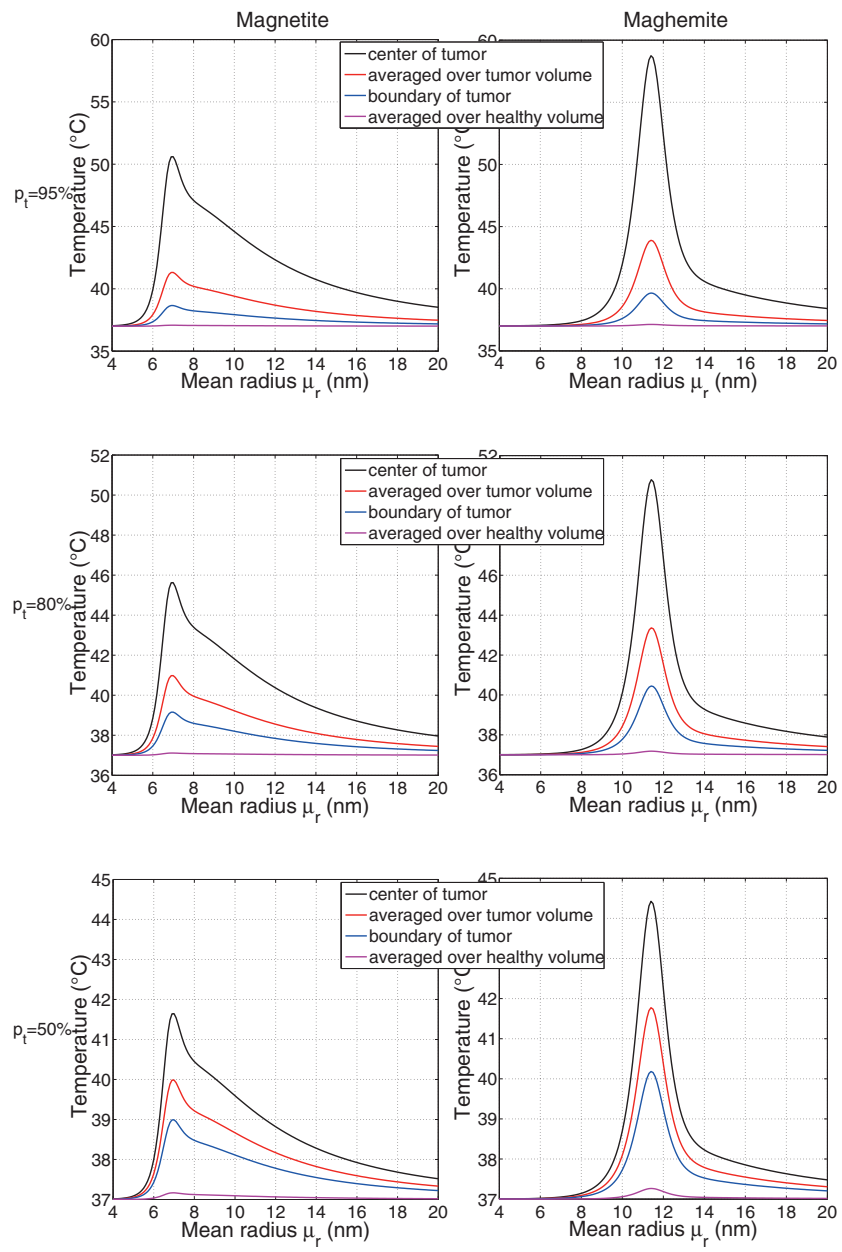

Fig. 3. Temperatures at $200 \mathrm{~s}$ when considering magnetite (left) and maghemite (right) magnetic nanoparticles with varying mean radius $\mu_{r}$. The standard deviation was fixed as $\sigma_{r}=0.01$. We varied the spatial spread of the magnetic nanoparticles inside the considered volume with varying percentage of magnetic nanoparticle inside the tumor, i.e. $p_{t}=95 \%, 80 \%, 50 \%$ in the upper, middle and lower subfigure, respectively. The temperature in the center of the tumor, temperature averaged over the tumor volume, temperature at the boundary of the tumor, and the temperature in healthy tissue (being a shell with inner radius of $6.2 \mathrm{~mm}$ and outer radius of $20 \mathrm{~mm}$ ) are shown.

polydisperse magnetic nanoparticle population.

\section{CONCLUSION}

This paper presents a numerical model for magnetic nanoparticle hyperthermia. The finite element model is able to calculate the spatio-temporal temperature distribution and the survival fraction of tumor cells. The linear response theory is used to determine the heating efficiency of superparamagnetic nanoparticles. We have additionally incorporated the effect of a log-normal particle size distribution for both magnetite and maghemite nanoparticles with their unique anisotropy constant and saturation magnetization. The influence of the spatial spread and thus the percentage of MNPs in the tumor was studied as well. The temperature rise significantly depends on the spatial spread of MNPs, indicating the importance of including the spatial distribution of MNPs. Finally, simulations were performed while varying the mean radius of magnetite and maghemite distributions. Again, the spatial spread plays 

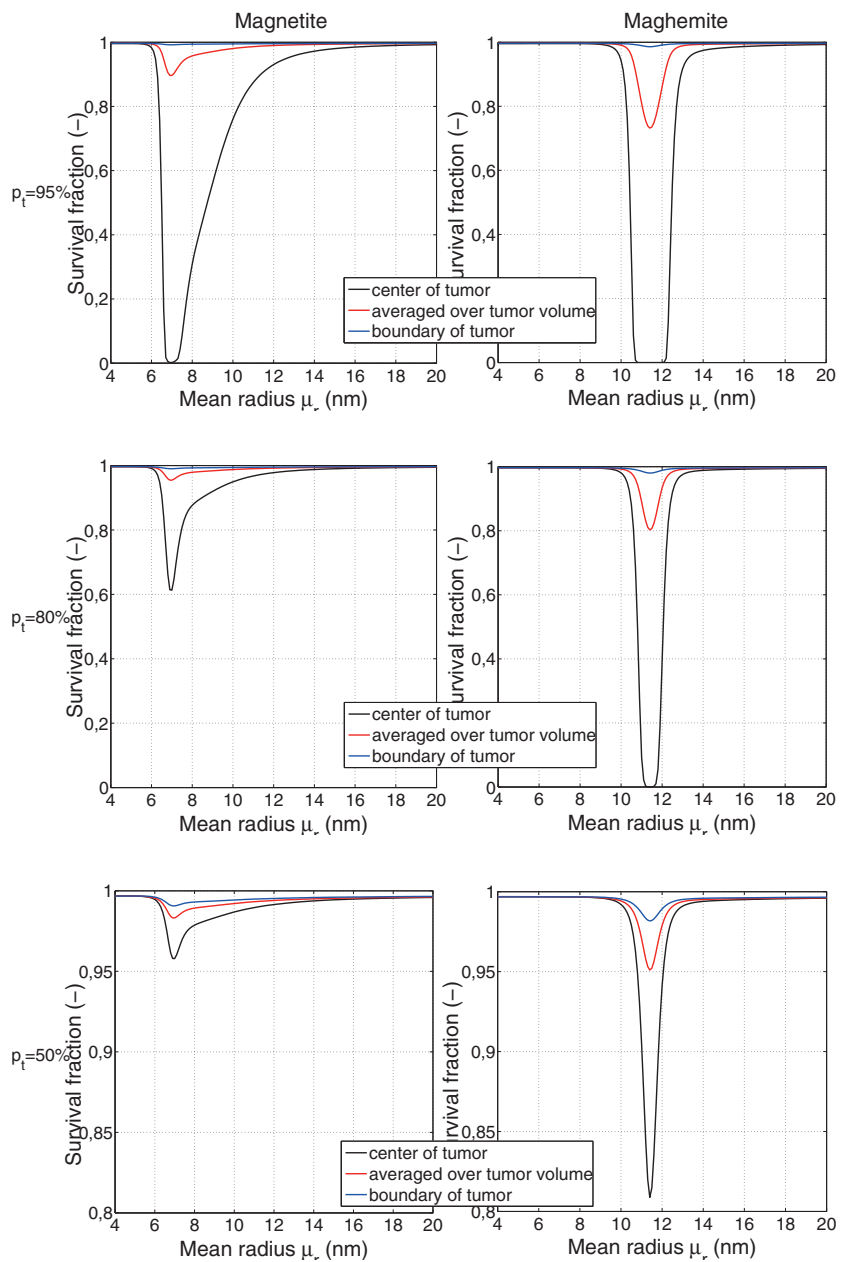

Fig. 4. Survival fractions at $200 \mathrm{~s}$ when considering magnetite (left) and maghemite (right) magnetic nanoparticles with varying mean radius $\mu_{r}$. The standard deviation was fixed as $\sigma_{r}=0.01$. We varied the spatial spread of the magnetic nanoparticles inside the considered volume with varying percentage of magnetic nanoparticle inside the tumor, i.e. $p_{t}=95 \%, 80 \%, 50 \%$ in the upper, middle and lower subfigure, respectively. The survival fraction in the center of the tumor, survival fraction averaged over the tumor volume, and survival fraction at the boundary of the tumor are shown.

a crucial role in obtaining therapeutic temperatures. Magnetite nanoparticles achieve a lower temperature rise than their maghemite counterparts, but therapeutic temperatures are obtained in a wider radius range. This computational model can be an important component in optimizing the superparamagnetic nanoparticle properties towards the therapeutical outcome.

\section{REFERENCES}

[1] R. Hergt, S. Dutz, R. Müller, and M. Zeisberger, "Magnetic particle hyperthermia: nanoparticle magnetism and materials development for cancer therapy," Journal of Physics: Condensed Matter, vol. 18, pp. S2919-S2934, 2006.

[2] P. Wust, B. Hildebrandt, G. Sreenivasa, B. Rau, J. Gellermann, H. Riess, R. Felix, and P.M. Schlag, "Hyperthermia in combined treatment of cancer," Lancet Oncology, vol. 3, pp. 487-497, 2002.

[3] C.L. Dennis, and R. Ivkov, "Physics of heat generation using magnetic nanoparticles for hyperthermia," International Journal of Hyperthermia, vol. 29, pp. 715-729, 2013.

[4] R. Rosenweig, "Heating magnetic fluid with alternating magnetic field," Journal of Magnetism and Magnetic Materials, vol. 252, pp. 370374, 2002.
[5] J. Carrey, B. Mehdaoui, and M. Respaud, "Simple models for dynamic hysteresis loop calculations of magnetic single-domain nanoparticles: Application to magnetic hyperthermia optimization," Journal of Applied Physics, vol. 109, pp. 083921, 2011.

[6] H.H. Pennes, "Analysis of tissue and arterial blood temperatures in the resting human forearm," Journal of Applied Physiology, vol. 1, pp. 93$122,1948$.

[7] B. Mehdaoui, A. Meffre, J. Carrey, S. Lachaize, L-M. Lacroix, M. Gougeon, B. Chaudret, and M. Respaud, "Optimal size of nanoparticles for magnetic hyperthermia: A combined theoretical and experimental study," Advanced Functional Materials, vol. 21, pp. 4573-4581, 2011.

[8] M.L. Etheridge, N. Manuchehrabadi, R.R. Franklin, and J.C. Bischof, "Superparamagnetic iron oxide nanoparticle heating," Nanoparticle heat transfer and fluid flow, CRC Press, 2012.

[9] F. Soetaert, L. Dupré, R. Ivkov, and G. Crevecoeur, "Computational evaluation of amplitude modulation for enhanced magnetic nanoparticle hyperthermia," Biomedizinische Technik/Biomedical Engineering, vol. 60, pp. 491-504, 2015.

[10] C. Geuzaine, and J.F. Remacle, "Gmsh: a three-dimensional finite element mesh generator with built-in pre- and post-processing facilities," International Journal for Numerical Methods in Engineering, vol. 79, pp 1309-1331, 2009.

[11] P. Dular, C. Geuzaine, F. Henrotte, and W. Legros, "A general environment for the treatment of discrete problems and its application to the finite element method," IEEE Transactions on Magnetics, vol. 34, pp. 3395-3398, 1998.

[12] J.A. Pearce, "Relationship between Arrhenius models of thermal damage and the CEM 43 thermal dose," Proceedings of the SPIE, vol. 7181, pp. $1-15,2009$.

[13] H. Hemala, J.S. Thakur, V.M. Naik, P.P. Vaishnava, G. Lawes, and R. Naik, "Investigation of magnetic properties of $\mathrm{Fe}_{3} \mathrm{O}_{4}$ nanoparticles using temperature dependent magnetic hyperthermia in ferrofluids," Journal of Applied Physics, vol. 116, Art. No. 034309, 2014. 